\title{
Análisis de la prevalencia de dientes supernumerarios y agenesia en pacientes con labio y paladar hendido atendidos en la Clínica Integral de Operación Sonrisa Honduras.
}

\section{RESUMEN}

El presente estudio, se realizó para determinar la prevalencia de dientes supernumerarios y agenesia en pacientes con labio y/o paladar hendido que asistieron a la Clínica Integral de labio y paladar hendido de Operación Sonrisa Honduras en agosto del año 2019.

El labio y/o paladar hendido (LPH) es una de las malformaciones congénitas estructurales más comunes; afecta el complejo cráneo facial y es el resultado de la falta de unión entre los procesos frontonasal, maxilar y medial nasal, y lateral.

Las anomalías dentales en pacientes con labio y/o paladar fisurado se presentan con mayor frecuencia que en el resto de la población, las cuales afectan ambas denticiones. Dentro de estas anomalías se observa la hipodoncia y la hiperdoncia que se encuentran más comúnmente alrededor del área fisurada (área del incisivo lateral). Asimismo, se presentan cambios en la posición, alteraciones en tamaño y forma de los dientes, defectos del esmalte, retraso en el desarrollo y erupción dental.

Las alteraciones que están relacionadas con el número de dientes como lo son las agenesias o hipodoncia y los supernumerarios o hiperdoncia, se producen en la etapa de iniciación del desarrollo de la dentición a nivel embrionario, y se encuentran asociadas a las mal oclusiones dentarias.

Este es un estudio comparativo y retrospectivo de corte transversal con una muestra de 150 pacientes con paladar hendido y expedientes con radiografía panorámica, de los cuales 83 pacientes fueron del sexo femenino representando el $55 \%$ y 67 pacientes fueron del sexo masculino representando el $45 \%$ de la población total.

El universo se basó en 31 (21\%) pacientes de los cuales presentaron dientes supernumerarios, 21 (14\%) Agenesia, 52 (34\%) no presen-

\section{Autores:}

Dra. (Inf.) Kathia Nicol Ferrufino Ávila ${ }^{1}$

Dr. Hernán Alejandro Álvarez Vijil 
taron dientes supernumerarios y 46 (31\%) presentaron ausencia de dientes supernumerarios y agenesia.

El tipo de hendidura palatina más afectada por dientes supernumerarios fue la fisura palatina unilateral izquierda con 22 pacientes.

PALABRAS CLAVES: Agenesia, supernumerario, labio y paladar hendido, anomalías dentales.

\section{Analysis of the prevalence of supernumerary teeth and agenesis in patients with cleft lip and palate treated at the Integral Clinic of Operation Sonrisa Honduras.}

\section{SUMMARY}

The following study carried out to determine the prevalence of supernumerary teeth and agenesis in patients with cleft lip and/or palate who attended the lip and cleft palate Integral Clinic of Operation Smile Honduras, until August 2019.

Cleft lip and/or palate (CLP) is one of the most common structural congenital malformations; it affects the craniofacial complex and is the result of the lack of union between the frontonasal, maxillary and medial nasal, and lateral processes.

Dental anomalies in patients with cleft lip and/or palate occur more frequently in comparison with the rest of the population, affecting both dentitions. Among these anomalies, noted hypodontia and hyperdontia, are most commonly found around the fissured area (lateral incisor area). In addition, changes in position, alterations in size and shape of teeth, enamel defects, delayed development and dental eruption.

Alterations related to the number of teeth, such as agenesis or hypodontia and supernumerary or hyperdontia, occur at the initial stage of dentition development at the embryonic period, and are associated with dental malocclusions.

This is a comparative and retrospective cross-sectional study with a sample of 150 patients with a cleft lip and/or palate and medical records with panoramic radiography, of which 83 patients were female, representing 55\% and 67 patients, were male, representing $45 \%$ of total population.

The population included in this trial consisted of $31(21 \%)$ patients with supernumerary teeth, $21(14 \%)$ agenesis, 52 (34\%) with no supernumerary teeth, and $46(31 \%)$ with an absence of supernumerary teeth and agenesis.

The kind of palatal cleft most affected by supernumerary teeth was the left unilateral palatal cleft with 22 patients.

KEY WORDS: Agenesis, supernumerary, cleft lip and palate, Dental anomalies. 


\section{Introducción}

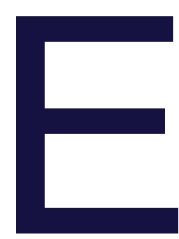

sta investigación surgió ya que existe la necesidad de ampliar los conocimientos en las diversas patologías dentales que se presentan en la Clínica de Operación Sonrisa Honduras; dado que existe muy poca referencia clínica y pocos estudios referentes a este tipo de patologías en el país, donde la inversión económica para el sector salud es limitada y la falta de conocimiento en el manejo clínico de las agenesias dentales y supernumerarios en pacientes con labio y paladar hendido en los centros de salud y las diferentes facultades de Odontología es una carencia.

Por medio del Departamento de Prótesis Facial y Maxilofacial, la Carrera de Técnico Universitario en Prótesis Bucal UNAH-CU y con vinculación a "Operación Sonrisa Honduras" se realizó la siguiente investigación. El labio y/o paladar hendido (LPH) es una de las malformaciones congénitas estructurales más comunes; afecta el complejo craneofacial y es el resultado de la falta de unión entre las procesos frontonasal, maxilar y medial nasal, y lateral. (P C. A., 2009) La dentición primaria se origina alrededor de la sexta semana del desarrollo embrionario, a partir de la odontogénesis que se define como el proceso embriológico que dará lugar a la formación del germen dental. (Cortés Paz, 2011).

Las anomalías dentales en pacientes con labio y/o paladar fisurado se presentan con mayor frecuencia que el resto de la población, afectado ambas denticiones. Dentro de estas anomalías se observa la hipodoncia y la hiperdoncia que se encuentran más comúnmente alrededor del área fisurada (área del incisivo lateral). Asimismo, se presentan cambios en la posición, alteraciones en tamaño y forma de los dientes, defectos del esmalte, retraso en el desarrollo y erupción dental. (Gomez, Fernández, \& Villanueva Vilchis, 2015).

Los pacientes con Labio y paladar hendido presentan ausencia congénita de uno o más dientes, esto se justifica puesto que al haber una deficiente unión de los procesos palatinos hay una alteración en la lámina dental que trae como consecuencia la ausencia en la formación del germen dentario, existiendo una relación directa entre la fisura y las anomalías. (Sáchez Peña., 2019).

Las alteraciones que están relacionadas con el número de dientes, como lo son las agenesias o 
hipodoncia y los supernumerarios o hiperdoncia, se producen en la etapa de iniciación del desarrollo de la dentición a nivel embrionario, y se encuentran asociadas a las mal oclusiones dentarias. (Guerra \& Valenzuela Rivera, 2014).

Las deformaciones del labio, alveolo y paladar hendido son malformaciones congénitas producidas por la no unión de algunos procesos faciales y que se presentan comúnmente en pacientes de todo el mundo, los cuales se encuentran en muchas investigaciones realizadas respecto a esta anomalía un promedio de incidencia de 1 en 1000 casos por año. (Tello Luis, 2008).

La incidencia de Fisura Labio palatina muestra diferente localización según el sexo, los varones con un $(60 \%)$; se afectan más que las mujeres (40\%) y tienden a presentar casos más graves. En las mujeres es algo más frecuente la fisura palatina aislada y en los varones, la completa y la anterior. (V. Gómez Clemente, 2017).

Según la organización Operación Sonrisa Honduras se estima que esta condición ocurre 1 de cada 500 recién nacidos. (Raudales, 2019).

Esta anomalía craneofacial afecta a los niños hondureños en diferentes aspectos como ser de autoestima, problemas psicosociales, dificultad en el habla, malnutrición, anomalías bucales, etc.
Esta investigación es de vital importancia ya que da a conocer la frecuencia de la agenesia dentaria y dientes supernumerarios en pacientes con labio y paladar hendido; dado que existe escasa evidencia y muy pocas publicaciones respecto al tema en Honduras.

Además, este estudio ayudará para tener una idea más clara de las estadísticas de estas anomalías y desde el punto de vista de rehabilitación oral, conocer la demanda de aparatología protésica al tener en cuenta los tratamientos para rehabilitar estos pacientes.

Los pacientes de labio y paladar se manejan bajo un protocolo clínico ortodóntico-ortopédico desde recién nacidos hasta edades adultas, los cuales necesitan en todo este proceso de aparatología ortopédica para mejorar su fonética, expansión de los segmentos maxilares buscando alineación del arco maxilar y según el caso específico de cada paciente hasta realizar extracciones de dientes supernumerarios y/o prótesis para reponer un diente con agenesia.

En el departamento de prótesis bucal y maxilo facial se propuso hacer esta investigación con el fin de conocer la prevalencia de las anomalías dentales de los pacientes y poder hacer alianzas con la organización "Operación Sonrisa Honduras", teniendo en cuenta la nueva carrera universitaria de téc- 
nico dental que ofrecerá la Universidad Nacional Autónoma de Honduras, para que los pasantes de dicha carrera elaboren los aparatos protésicos que la organización solicite.

\section{Tipo de Estudio}

Comparativo, retrospectivo de corte transversal, en este estudio se estimará la prevalencia de dientes supernumerarios y agenesia en pacientes con labio y paladar hendido.

\section{Universo}

Se tomaron en cuenta expedientes con radiografía panorámica de pacientes de 7 a 51 años con Paladar hendido unilateral completo, bilateral, hendidura de paladar blando, hendidura de paladar duro y hendidura alveolar que acudieron a la clínica Odontológica de Operación Sonrisa Honduras para recibir tratamiento desde 2009 hasta agosto 2019.

\section{Muestra}

Se seleccionó una muestra de 150 historias clínicas (Femenino y Masculino) con el fin de revisar y analizar radiografías panorámicas pertenecientes a pacientes con fisura alveolo palatina tomadas entre los 7 y 51 años.

Se trabajó con muestra aleatoria.

\section{Unidad de análisis}

Pacientes con Paladar hendido unilateral completo, bilateral, hendidura de paladar blando, hendidura de paladar duro y hendidura alveolar con expedientes con radiografías panorámicas.

\section{Lugar Seleccionado}

Clínica Integral de Labio y $\mathrm{Pa}$ ladar Hendido, Operación Sonrisa Honduras.

\section{Variables}

Pacientes con hendidura palatina unilateral completa, hendidura palatina bilateral, hendidura alveolar, hendidura de paladar blando, hendidura de paladar duro, pacientes sin dientes supernumerarios, dientes supernumerarios, agenesia, edad y sexo.

\section{Criterios de Inclusión}

Pacientes de 7-51 años con hendidura palatina unilateral completa, bilateral, hendidura de paladar blando, hendidura de paladar duro.

\section{Resultados}

Los resultados del presente estudio se obtuvieron por medio de los expedientes clínicos de "Operación Sonrisa Honduras" en el que 38 se encuentran: datos personales de los pacientes, tipo de hendidura, presencia de dientes supernumerarios, presencia de agenesia y 
ausencia de ambas patologías.

Se revisaron 150 expedientes de los pacientes en los cuales solo se incluyeron aquellos pacientes que presentaran hendidura palatina con expedientes que contaran con radiografías panorámicas. Se revisaron los expedientes de la siguiente manera: expedientes de pacientes con hendidura palatina (unilateral derecho, unilateral izquierdo, bilateral, alveolar, de paladar blando, de paladar duro) con presencia de supernumerarios (mesiodens, parapremolar, paramolar, distomolar) y/o agenesia, y ausencia de estas anomalías.

\section{Análisis de los Resultados}

Al realizar el estudio sobre la prevalencia de dientes supernumerarios y agenesia en pacientes con labio y paladar hendido que han asistido a la clínica integral de labio y paladar hendido de "Operación Sonrisa Honduras", hasta agosto 2019.

Al realizar la comparación según tipo la hendidura se observó que el $37.3 \%$ presenta hendidura palatina unilateral izquierda con relación a los demás tipos de fisuras palatinas con mayor prevalencia de dientes supernumerarios en posición mesiodens y mayor prevalencia de agenesia. Los pacientes con fisura palatina unilateral derecha son los segundos con más prevalencia de anomalías dentarias. (Figura 1).
-Los pacientes con hendidura de paladar duro y alveolar, no presentaron anomalías dentarias como dientes supernumerarios $\mathrm{y} / \mathrm{o}$ agenesias.

-Los pacientes con hendidura bilateral presentan prevalencia de 26 dientes sin anomalías dentarias (45\%), 20 presentan agenesia (34\%) y solamente 12 dientes supernumerarios $(21 \%)$.

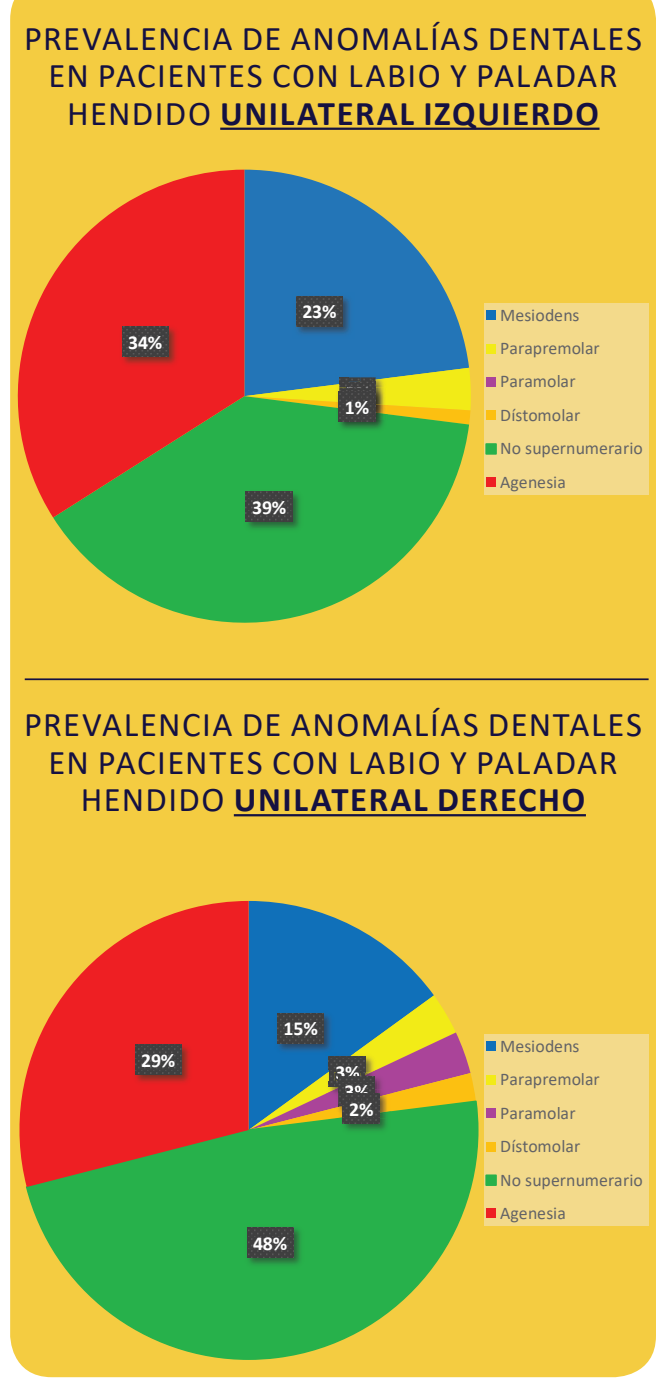

Figura No.1 Comparación de prevalencia de anomalías dentales en hendidura unilateral derecha e izquierda. 
-Las hendiduras palatinas de paladar blando y duro son de menor prevalencia y sus anomalías dentarias son menores en comparación a los demás tipos de fisura.

-En la muestra revisada de este estudio se observó una mayor prevalencia de labio y paladar hendido unilateral izquierdo en el sexo femenino (55\%) que en el masculino (45\%). (Figura 2).

Una diferencia según algunos estudios realizados está la malformación craneofacial esta es la más frecuente en el sexo masculino. Dicha anomalía puede estar ligada a factores genéticos y ambientales.

-Al revisar las prevalencias de dientes supernumerarios en la hendidura distribuidos según sexo se encontró que es mayor en las pacientes femeninas que los masculinos. Por tanto, se rechaza la hipótesis que la prevalencia de anomalías dentarias es mayor en hombres que en mujeres.

- En la muestra revisada de este estudio se observó una mayor prevalencia de agenesia y no supernumerarios (31\%) que dientes supernumerarios $(21 \%)$; ya que con los resultados obtenidos se rechaza la hipótesis que los dientes supernumerarios tienen mayor prevalencia que las agenesias en pacientes con paladar hendido. (Figura 3).

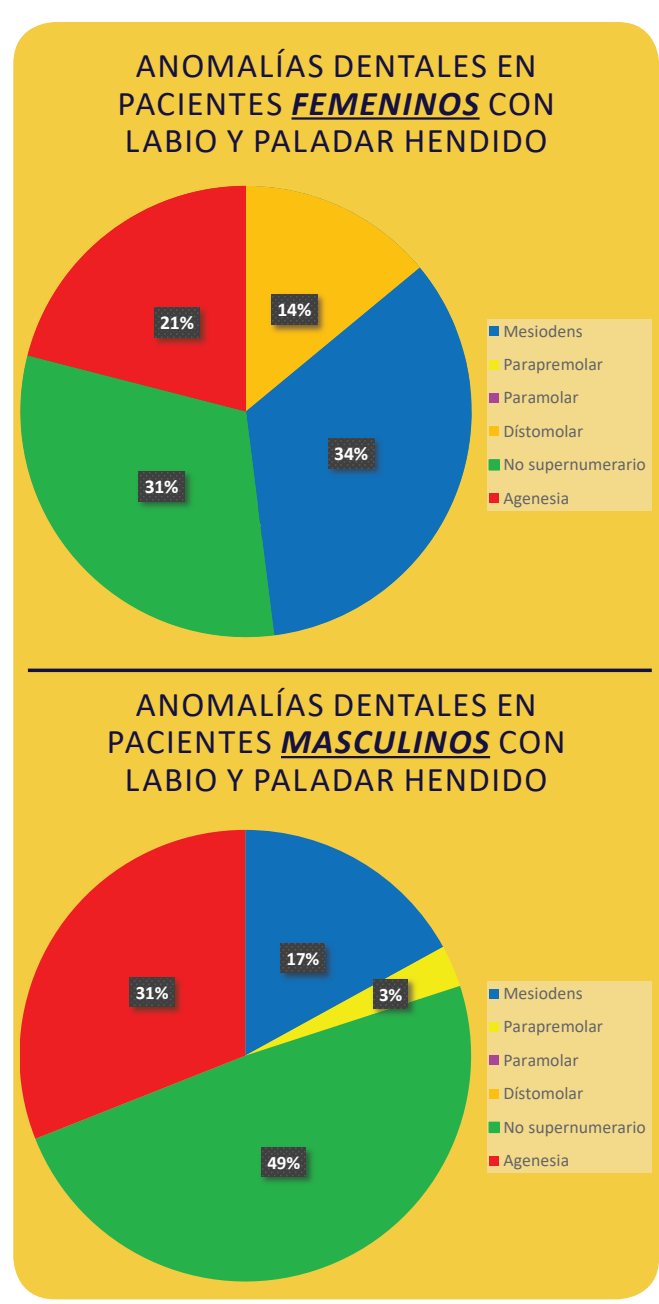

Figura No.2 Comparación de anomalías dentales en pacientes femeninos y masculinos con LPH.

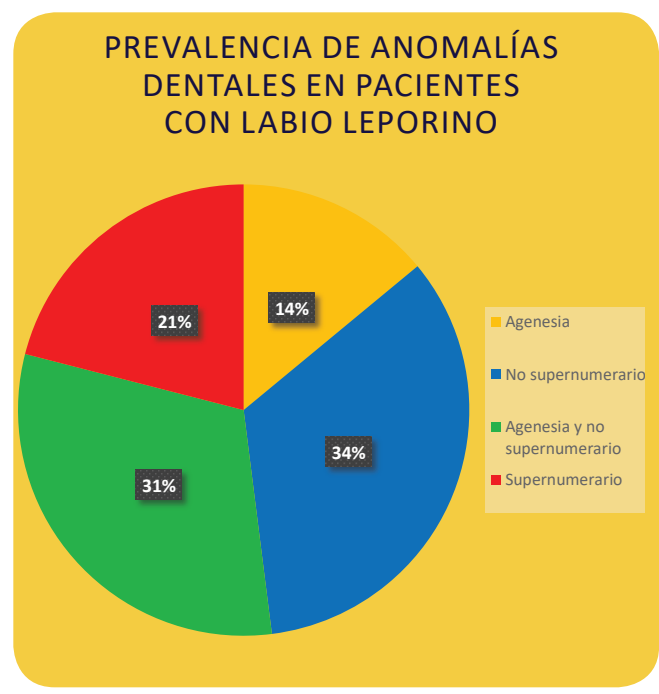

Figura No.3 Resumen de prevalencia de anomalías dentales en pacientes con LPH. 

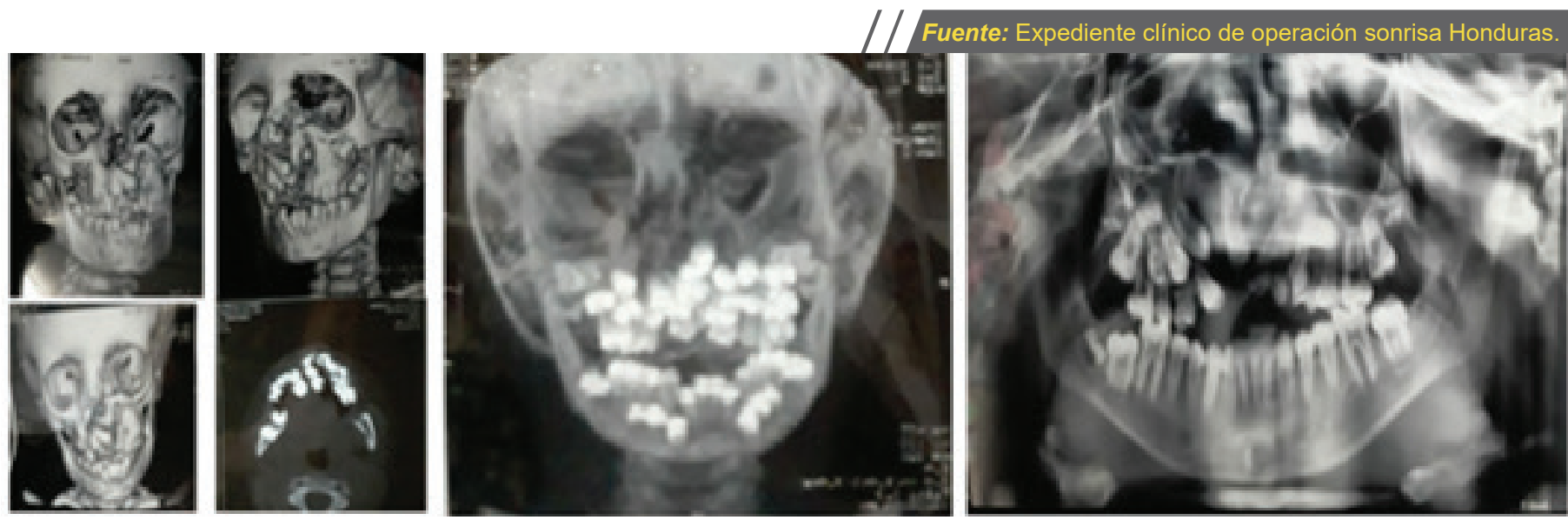

Figura No.4 Radiografías de paciente con múltiples dientes supernumerarios. A) Tomografía computarizada B) Radiografía panorámica post-quirúrgica

\section{Conclusiones}

1. Los pacientes de "Operación Sonrisa Honduras" con el uso de aparatología instalada luego del tratamiento de sus agenesias mostraron una mejoría en su autoestima y desarrollo personal, además, de incrementar una mejoría en fonética (terapia de lenguaje) y una mejor eficacia masticatoria

2. El tratamiento de un diente supernumerario depende del tipo y posición de este y de su efecto potencial sobre los dientes adyacentes.

3. El tratamiento de los dientes supernumerarios depende de cada caso en particular, el cual varía desde observación y extracción, hasta cirugía para su remoción.

4. Los pacientes con hendidura de paladar duro y alveolar, no presentaron anomalías dentarias como dientes supernumerarios y/o agenesias.

5. Se encontró mayor prevalencia de dientes supernumerarios $y$ agenesia en los pacientes de sexo femenino que en los masculinos.

6. Se obtuvo resultados que señalan mayor número de agenesias que dientes supernumerarios.

7. Por medio de la Facultad de Odontología y el Técnico Universitario en Prótesis Bucal y con vinculación a (Operación Sonrisa Honduras) se realizarán los aparatos ortopédicos para los pacientes que asisten a la clínica integral de "Operación Sonrisa Honduras". 


\section{Recomendaciones}

1. El diagnóstico temprano es importante para el buen manejo del paciente, debido a que permite escoger la más apropiada opción de tratamiento según las necesidades de este para minimizar futuras complicaciones.

2. Desde el punto de vista profesional, sugerir siempre a los profesionales de la salud en este caso a los odontólogos a que se actualicen en cuanto al manejo integral de los pacientes con labio y paladar hendido para así estar capacitados en cualquier momento cuando este tipo de casos se nos presenten.

3. A la Facultad de Odontología, se sugiere enviar a pasantes de Servicio Social de cada generación a dicho centro para poder continuar con la labor de atención a los niños y jóvenes, ya que se obtiene un gran aprendizaje en el manejo y plan de tratamiento de estos pacientes, al igual que se agudiza el espíritu de voluntariado y ayuda a los más necesitados.

4. A la organización "Operación Sonrisa Honduras", se sugiere educar a los pacientes acerca de la importancia que tiene la salud dental y conocer sobre estas anomalías para evitar miedos y estrés innecesarios tanto a los padres como a los pacientes. Y sobre todo para que comprendan la importancia de llevar a cabo un plan integral sin desesperarse para obtener resultados finales más efectivos.

\section{Responsabilidades éticas}

Protección de personas y animales. La autora declara que los procedimientos seguidos se conforman a las normas éticas del comité de experimentación humana responsable y de acuerdo a la Asociación Médica Mundial.

Confidencialidad de los datos. La autora declara que ha seguido los protocolos de su centro de trabajo sobre la publicación de datos de pacientes.

\section{Derecho a la privacidad y con-} sentimiento informado. La autora declara que en este artículo no aparecen datos personales de pacientes. 


\section{Bibliografía}

Gómez, Olimpia Viguera; Fernández, Miguel Ángel; Villanueva Vilchis, María del Carmen, Prevalencia de dientes supernumerarios en niños con labio y/o paladar fisurado, Revista odontológica mexicana, (2015), páginas 81-88.

Guerra, Irene Gutiérrez; Valenzuela Rivera, Oriana, Alteraciones del número en dentición de pacientes entre 2 y 12 años de edad con disrafias labio alveolo palatina atendidos en la unidad de odontopediatría del Hospital Regional Antofagasta,Chile, Revista Int. J. Odontostomat, (2014) Páginas 481-490.

Camilo Andrés Serrano, LABIO YIO PALADAR HENDIDO: UNA REVISIÓN, Páginas 44-52. (2009), 01 de julio. file://C:/Users/Eduard/Downloads/11802586-1-SM.pdf

Cortés Paz Marta, Maduración y desarrollo dental de los dientes permanentes en niños de la comunidad de Madrid. Aplicación a la estimación dentaria. Madrid (2011).Recuperado de https://www.google.com/ url?sa=t\&source=web\&rct=j\&url=https://eprints.ucm.es/19916/1/Marta_Paz_Cort\%25C3\%25A9s-trabajo_de_investigaci\%25C3\%25B3n..pdf\&ved=2ahUKEwic9fKhwaPsAhUmw1kKHTajBesQFjAKegQIAxAB\&us-

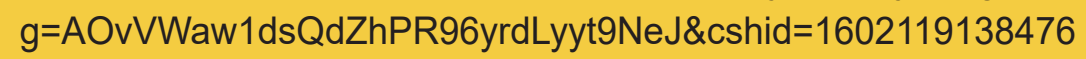

Tello Luis, Paricoto Olinda. Prevalencia de anomalías dentarias en pacientes con fisura labio alveolo palatina atendidos en el Instituto Especializado de Salud del Niño. Lima, Perú. Odontología Sanmarquina 2008; 11(2): 56-59. Recuperado de https://www.researchgate.net/scientific-contributions/2114501611-Luis-Alberto-Mogollon-Tello

V. Gómez Clemente, J. López- Garco Torres, A. Macías Gago. I. Nieto Sánchez, L. Aneiros Fernández. Protocolo Ortopédico_Ortodóntico de Actuación en Pacientes con Fisura Labio-Alveolar y palatina. Odontol Pediátr (Madrid) Vol.25, №3, pp.173-190,2017. Recuperado de https://www. google.com/url?sa=t\&source=web\&rct=j\&url=https://www.odontologiapediatrica.com/wp-content/uploads/2018/05/338_03_Original_318_Gomez. pdf\&ved=2ahUKEwiGhOW6t6PsAhVIpFkKHVbIB1U4ChAWMAR6BAgEEAE\&usg=AOvVaw3dNuCq8LjtV8ATtUBXOLoQ

Raudales, Anny, Eficiencia de la placa modeladora sobre la disminución de la amplitud de la hendidura labial unilateral en niños de 0-6 meses con terapia de tracción labial utilizando como comparación a los niños de 0-6 meses que solo están en terapia de tracción labial, 2019.

Sánchez, Peña, M. K., \&Galvis Aricapa, JA. (2019). Anomalías dentales de los pacientes con labio y paladar hendido: revisión de la literatura. Revista Nacional de odontología. (2019) ,1-17. Recuperado de: https://do i,org/10.16925/2357-4607.2019.02.01 